\title{
Patency and complications of patients with tunneled dialysis catheters: A long-term follow-up study
}

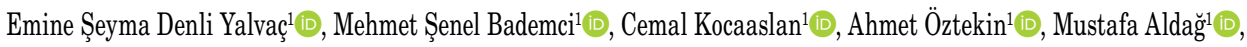

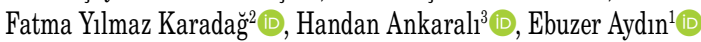 \\ ${ }^{1}$ Department of Cardiovascular Surgery, Istanbul Medeniyet University, Göztepe Training and Research Hospital, Istanbul, Turkey \\ ${ }^{2}$ Department of Infectious Diseases, Istanbul Medeniyet University, Göztepe Training and Research Hospital, Istanbul, Turkey \\ ${ }^{3}$ Department of Biostatistics and Medical Informatics, Istanbul Medeniyet University, Göztepe Training and Research Hospital, Istanbul, Turkey
}

\begin{abstract}
Objectives: This study aims to report long-term follow-up results including catheter patency and catheter dysfunction in patients with tunneled dialysis catheters (TDCs) during the treatment phase.

Patients and methods: Medical records of a total of 90 patients (54 males, 36 females; mean age $59.9 \pm 19$ years; range, 19 to 89 years) with a combined total of 103 TDCs inserted by a single cardiovascular surgeon at our institution between January 2014 and December 2017 were reviewed retrospectively. Data including age, gender, duration of hospital stay, catheter patency, complications, and mortality were recorded. The patients were divided into two groups according to age: $<60$ years $(n=41)$ and $\geq 60$ years $(n=49)$.

Results: Technical success was achieved without any procedural complications and hemodialysis was initiated following the catheter placement in all patients. The mean duration of catheter use was $729.6 \pm 58.4$ (range, 20 to 1,100) days. Of all patients, 18 (20\%) died within one year following the procedure. A total of 28 TDCs (24.77\%) were electively removed, as these patients gained other types of permanent access. One of them underwent a kidney transplant. Catheter-related complications were observed in 24 patients (21.23\%) and TDCs of 13 patients were replaced. Twelve complications (10.61\%) were related to catheter dysfunction, and the remaining 12 were due to catheterrelated infections (CRIs). Ten of the patients with CRIs were treated successfully, while the remaining two died. No significant correlation was found between the catheter patency, catheter-related complications, and baseline characteristics of the patients, except for age. Younger age was a predictor of poor catheter patency $(p=0.040)$. Fifty six patients $(62 \%)$ who were discharged on the day of their catheter placement had significantly fewer complications than the hospitalized patients $(\mathrm{p}=0.011)$.

Conclusion: The prevention of TDC-related complications requires a careful insertion technique and extensive surgical experience. The TDC patency and reducing the number of complications are also closely associated with early discharge and the age of patient.

Keywords: Catheter patency, catheter-related complication, catheter-related infection, long-term follow-up, surgical experience, tunneled dialysis catheter.
\end{abstract}

The number of patients with end-stage renal disease (ESRD) has been currently increasing worldwide. ${ }^{[1]}$ This increase has also increased the unmet need for permanent vascular access procedures for these patients. Permanent vascular access is provided by a tunneled (cuffed) dialysis catheter (TDC), when no appropriate vessels are accessible for an arteriovenous fistula (AVF) or arteriovenous graft (AVG). ${ }^{[1]}$ The TDCs can be placed by surgeons, interventional radiologists, or nephrologists. The use of TDCs often leads to increased complications and mortality. ${ }^{[2]}$ The internal jugular veins (IJVs), external jugular veins, femoral veins, subclavian veins (SCVs), and transhepatic accesses and translumbar accesses to the inferior vena cava can be selected for the placement of a TDC. ${ }^{[3]}$ The right IJV is the most preferred vascular access site for TDCs due to its technically simple accessibility and its unhindered, straight passage to the right atrium according to The National Kidney Foundation Kidney Disease Outcomes Quality

Received: February 12, 2019 Accepted: April 05, 2019 Published online: July 25, 2019

Correspondence: Ebuzer Aydın, MD. İstanbul Medeniyet Üniversitesi Göztepe Eğitim ve Araştırma Hastanesi Kalp ve Damar Cerrahisi Kliniği, 34722 Kadıköy, İstanbul, Turkey. e-mail: drebuzeraydın@gmail.com 
Initiative (NKF KDOQI) ${ }^{[3]}$ Of note, a femoral access for a TDC is not preferred due to the increased risk of infection and occlusion. ${ }^{[4]}$ The SCV is also less preferred for placing a TDC, as it is associated with an increased risk of venous stenosis. ${ }^{[5]}$

In general, TDCs are applied according to the modified Seldinger method and the use of portable high-resolution real-time ultrasound devices percutaneously. ${ }^{[6]}$ Procedural and late complications are two forms of TDC complications. The use of real-time ultrasound-guided cannulation decreases the risk of procedural complications, such as arterial puncture, pneumothorax, hemothorax, and misplacement. ${ }^{[7]}$ Infections and catheter dysfunction are the most common examples of late complications which may lead to catheter removal, if they are not successfully treated. ${ }^{[8]}$ Catheter dysfunction develops due to a catheter lumen thrombus, fibrin sheath formation, catheter migration, or stenosis of the involved vein, unless an extracorporeal blood flow of $300 \mathrm{~mL} / \mathrm{min}$ is achieved. ${ }^{[9]}$

Catheter lumen thrombosis is the most common cause of catheter dysfunction with a high incidence up to $46 \% .^{[2]}$ The most common cause of hospitalization after TDC catheterization is catheter-related infections (CRIs), which can occur in two ways: through the lumen or around the catheter. ${ }^{[10]}$ Catheter-related infections are seen in about one-fifth of patients. ${ }^{[10-12]}$ The most significant risk factor for a CRI is the duration that the catheter is used. ${ }^{[9]}$ Localization of the catheter, immunosuppression, and diabetes mellitus (DM) are also considered to be other risk factors for CRI. ${ }^{[9]}$ Catheter-related bacteremia (CRB) is associated with positive blood cultures in patients with signs and symptoms of bacteremia. ${ }^{[12]}$ Finding of a positive catheter culture, if available, with the same organism as seen on a blood culture supports the diagnosis of CRB. Catheter dysfunction, CRB (despite appropriate antibiotic treatment for at least two days, hemodynamic instability, and evidence of metastatic infections are common reasons that TDCs are removed.

In the present study, we aimed to present our long-term results and complications of TDCs placed by a single cardiovascular surgeon at our institution.

\section{PATIENTS AND METHODS}

Medical records of a total of 90 patients (54 males, 36 females; mean age $59.9 \pm 19$ years; range, 19 to 89 years) with a combined total of 103 TDCs inserted by a single cardiovascular surgeon at Istanbul Medeniyet University, Göztepe Training and Research Hospital between January 2014 and December 2017 were reviewed retrospectively. Only those who received a TDC for hemodialysis by our department and patients aged $\geq 18$ years were included in this study. Those who received TDCs for a purpose other than hemodialysis and those in whom TDCs were placed by other departments were excluded. A written informed consent was obtained from each patient. The study protocol was approved by Istanbul Medeniyet University, Göztepe Training and Research Hospital Ethics Committee (2018/0084). The study was conducted in accordance with the principles of the Declaration of Helsinki.

Data including age, gender, duration of hospital stay, catheter patency, complications, and mortality were recorded. In addition, whether the patients were discharged on the placement day was evaluated. One hundred and three TDCs were placed through the right IJV ( $\mathrm{n}=84)$, left IJV ( $\mathrm{n}=15)$, and femoral vein $(n=4)$. Twenty-eight of these patients were waiting for AVF maturation and 62 of these patients did not have an appropriate vessel for access. All patients were divided into two groups according to age: $<60$ years $(n=41)$ and $\geq 60$ years $(n=49)$.

\section{Tunneled dialysis catheter placement technique}

All TDCs were placed percutaneously under sterile conditions by a single cardiovascular surgeon under real-time ultrasound (SonoScape S40 Color Doppler Ultrasound System, Guangdong, China) in an operating room. Local anesthesia was applied to all patients. Double-lumen permanent hemodialysis catheters (Permcath ${ }^{\mathrm{TM}}$, Covidien, Mansfield, Massachusetts, USA) made of a soft silicone material with a length of $19 \mathrm{~cm}$ for the right IJV, 23 to $28 \mathrm{~cm}$ for the left IJV, and $28 \mathrm{~cm}$ for the femoral vein were preferred. The TDCs were tunneled subcutaneously at least $10-\mathrm{cm}$ from the vein entry site to reduce the risk of bacteremia. Each TDC was controlled with its tip in the superior vena cava under fluoroscopy. No antibiotic prophylaxis was applied before the procedures.

\section{Definitions}

Technical success was defined as the patient having at least one hemodialysis session without any procedural complications. ${ }^{[9]}$ Catheter dysfunction was defined as when a patient's blood flow rate was under $300 \mathrm{~mL} / \mathrm{min} \cdot{ }^{[13]}$ Catheter exit site infection was defined as when pericatheter erythema and/or purulent drainage $<2-\mathrm{cm}$ away from the catheter exit 
site without positive blood cultures occurred. ${ }^{[12]}$ A tunnel infection was defined as when erythema and purulent drainage occurred $>2-\mathrm{cm}$ away from the catheter exit site without positive blood cultures. Catheter-related bacteremia was defined as when systemic signs and symptoms of infection, such as fever or chills, in combination with positive blood cultures, were observed. Catheter patency (catheter survival) was the amount of time between the TDC placement and the TDC removal due to death, complications, or lack of need.

\section{Statistical analysis}

Statistical analysis was performed using the IBM SPSS version 23.0 software (IBM Corp., Armonk, NY, USA) and Wizard Pro 1.9.19 for MacOS $\left({ }^{\circledR} 2008-2017\right.$ Evan Miller). Descriptive statistics were expressed in mean \pm standard deviation (SD), range (min-max), or number and frequency. Quantitative data were analyzed using the Kolmogorov-Smirnov test for normality assumption, and skewness and kurtosis values of distribution were calculated. Differences between the right and left measurements were compared using the paired samples t-test. A 95\% confidence interval (CI) was used. The relationship between age and ratio was investigated using the Spearman rank correlation analysis. The independent samples t-test was used to compare the sexes in terms of ratio. Catheter patency was calculated using the Kaplan-Meier method. After age categorization, Cox regression modelling and the log-rank test were used to evaluate age groups and gender differences in terms of catheter patency. A $p$ value of $<0.05$ was considered statistically significant.

\section{RESULTS}

Baseline demographic and clinical characteristics of the patients are summarized in Table 1 . Thirty-four patients $(37.7 \%)$ were hospitalized and 56 patients

\begin{tabular}{lccc}
\multicolumn{4}{l}{ Table 1. Baseline characteristics of patients $(\mathbf{n}=\mathbf{9 0})$} \\
\hline Characteristics & $\mathrm{n}$ & $\%$ & Mean \pm SD \\
\hline Age (year) & & & $59.9 \pm 19$ \\
Gender & 54 & 60 & \\
$\quad$ Male & & & \\
Insertion site & 84 & 74.33 & \\
$\quad$ Right IJV catheters & 15 & 13.27 & \\
$\quad$ Left IJV catheters & 0 & 0 & \\
$\quad$ Subclavian catheters & 4 & 3.53 & \\
$\quad$ Femoral catheters & 56 & 62.2 & \\
Discharged & 34 & 37.7 & \\
Hospitalized patient &
\end{tabular}

(62.2\%) were discharged on the day of placement. No procedural complications, such as pneumothorax or air embolism, were observed in any of the patients. The outcomes of the TDCs during follow-up are summarized in Table 2.

Seventy-seven patients (85.55\%) were given one TDC, and 13 patients (14.4\%) were given two TDCs. Overall, the TDCs of 28 patients (31.11\%) were removed, as these patients gained other types/sites of permanent access. Of these, one patient received a kidney transplant. Catheter-related complications were observed in 24 of the patients under two main headings: catheter dysfunction and CRI. Catheter dysfunction was found in 12 of these 24 patients (13.33\%), and the TDCs of five of them were exchanged over a guidewire with the creation of a new tunnel. Twelve patients with complications had CRIs. Of these patients, CRB, with a positive blood culture rate, was reported in eight (8.88\%). In these patients, the causative microbiological agents were methicillinsensitive staphylococcus aureus (MSSA), methicillinresistant staphylococcus aureus (MRSA), klebsiella pneumoniae, staphylococcus hominis, enterococcus spp., and pseudomonas spp.

Overall, 18 patients (20\%) died within one year, two of whom (11.11\%) due to CRIs. Of the total number of TDCs, $19.41 \%$ were removed due to catheter-related complications. Fifty-six patients were discharged on the day of catheter placement, and these patients experienced significantly fewer complications than the hospitalized patients $(\mathrm{p}=0.011)$. The mean duration of catheter use was $729.6 \pm 58.4$ (range, 20 to 1,100) days (Figure 1). The patients under 60 years of age $(n=41)$ had a mean catheter patency of $875.6 \pm 66.9$ days, and the patients over $60(n=49)$ had a mean catheter patency of $613.2 \pm 80.7$ days. Gender was not a significant factor

\begin{tabular}{lcc} 
Table 2. Outcomes of TDCs during follow-up (n=103) \\
\hline Catheter status & $\mathrm{n}$ & $\%$ \\
\hline Functioning catheter & 38 & 36.89 \\
Functioning catheter with no complication & 20 & 19.41 \\
Functioning catheter at death & 18 & 17.47 \\
Elective removal & 28 & 27.18 \\
Transplanted & 1 & 0.97 \\
Removed due to all complication & 24 & 23.3 \\
Removed due to catheter infection & 12 & 11.65 \\
Exit-site infection & 2 & 1.94 \\
Tunnel infection & 2 & 1.94 \\
Bacteremia & 8 & 7.76 \\
Removed due to catheter thrombosis & 12 & 11.65 \\
\hline TDC: Tunneled (cuffed) dialysis catheter. & &
\end{tabular}




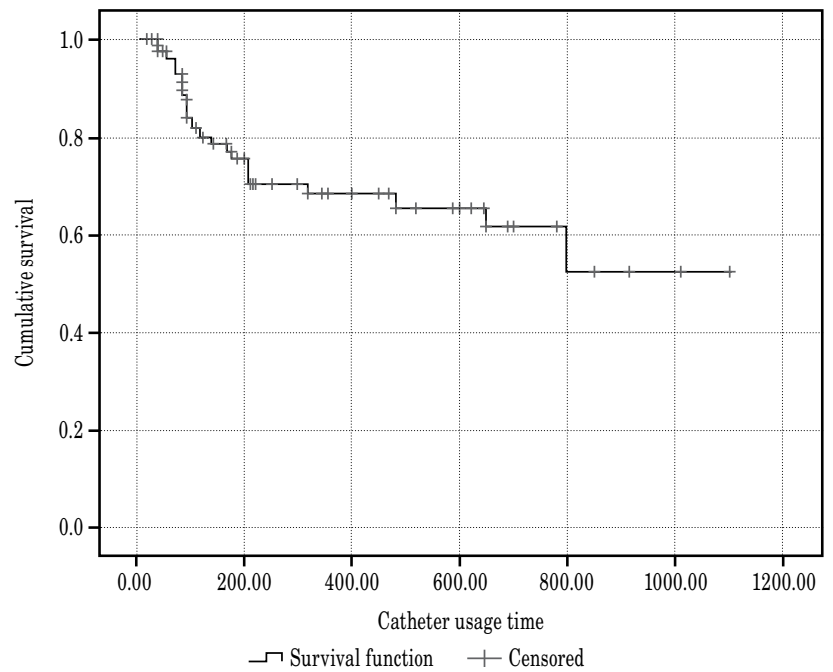

Figure 1. The mean time of catheter use.

among the patients with catheter-related complications and poor catheter patency $(\mathrm{p}=0.382)$. However, catheter patency was significantly related to the patient age $(\mathrm{p}=0.040)$ (Figure 2).

\section{DISCUSSION}

Despite high complication and mortality rates, TDC placement still remains an effective option for vascular access in patients with ESRD. Patients with hemodialysis catheters dissatisfy with living with these catheters, although they cannot survive without them. ${ }^{[14]}$ Coentrao et al. ${ }^{[15]}$ reported that the one-year survival rate of patients with TDCs was lower than other types of vascular access. The present study was unable to evaluate survival rate in other hemodialysis patients; however, it exhibited a mortality rate up to $20 \%$. Thus, we cannot conclude that it is the highest rate, while it is not low.

In our study, late complications were observed; however, we found no procedural complications such as arterial puncture, pneumothorax, hemothorax, or misplacement during the catheter placement. Therefore, we conclude that surgical experience of physicians and ultrasound guidance significantly reduced the number of procedural complications, as Bagul et al. ${ }^{[2]}$ reported. In addition, in our study, no infection problems emerged regarding the catheter placement technique, as all CRIs occurred long after the time of insertion. In general, it is thought that the TDC is cuffed to provide a physical barrier to infection; however, the infection rate is still quite high. Overall, although it is considered that TDC

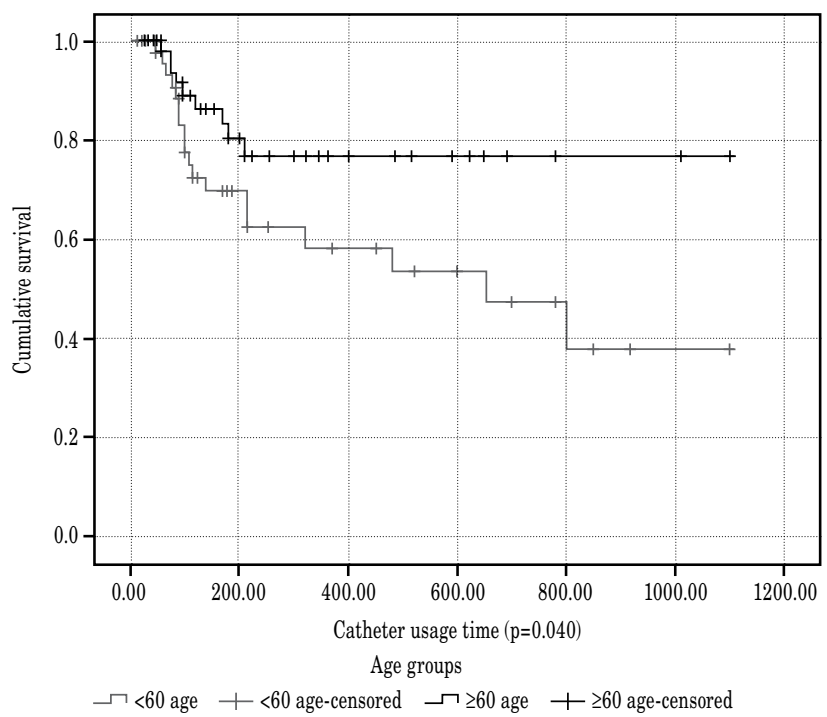

Figure 2. The catheter patency relationship with patient age $(\mathrm{p}=0.040)$.

has a cuffed to provide physical barriers to infection, infection rate is not very low. Infections following the long-term use of a catheter are usually seen in 19\% of patients; $7 \%$ are considered local infections, and $12 \%$ are considered CRB. ${ }^{[11]}$ In their study, Maki et al. ${ }^{[16]}$ reported that the incidence of $\mathrm{CRB}$ varied between 0.1 and $22.5 \%$. In our study, CRIs were observed in only 12 patients $(13.3 \%$ of the total number of patients and $11.6 \%$ of the total number of TDCs), and we found an $8.8 \%$ incidence of CRB which is significantly lower than those reported in previous studies.

Furthermore, DM has been shown to be associated with catheter-related complications in the literature. ${ }^{[9,17]}$ However, clinical databases containing hospital records do not include whether patients have diabetes; thus, DM was not taken into consideration in our study. However, it is noteworthy to mention that several studies have shown that DM is not associated with catheter-related complications. ${ }^{[18]}$ Although TDC removal rates ranging from $32^{[19]}$ to $39.6 \%{ }^{[20]}$ have been reported due to catheter-related complications, in our study, $19.41 \%$ of TDCs were removed, which is significantly lower than the literature.

Considering the duration of catheter use, Shingarev et al. ${ }^{[21]}$ reported a mean of 202 days, Fry et al. ${ }^{[19]}$ reported a mean of 506 days, and Golebiowski et al. ${ }^{[22]}$ reported a mean of $354 \pm 422$ days. In our study, the mean duration of catheter use was $729.6 \pm 58.4$ days, which is significantly higher than the literature. There can be several reasons for our favorable finding related 
to functioning duration of TDCs. One reason could be that the majority of our patients were discharged on the day of catheter placement and these patients had significantly less complications than the hospitalized patients $(\mathrm{p}=0.011)$. According to a previous study (Salonen and Saha, 2014), ${ }^{[23]}$ however, the patients who were discharged on the day of catheter placement had similar outcomes to the hospitalized patients. Fry et a1. ${ }^{[19]}$ also reported that age, sex, and specialty of physician (surgeon or clinician) did not significantly affect the catheter patency. In another study, Lund et al. ${ }^{[20]}$ compared the outcomes of TDCs which were placed by interventional radiologists with those placed by surgeons, and obtained similar outcomes. Therefore, the reason for higher values in our study may be due to the placement of the TDCs which was performed by a cardiovascular surgeon. Surgeons are familiar with all the precautions that must be taken for surgical skin preparation in the operating room and, particularly, cardiovascular surgeons are the most likely to be experienced on how to place a TDC into any vein. Nonetheless, based on our study results, it is surprising that patients over 60 years of age were associated with significantly higher catheter patency. Kocaaslan et al. ${ }^{[24]}$ reported that elderly patients who developed AVFs also had a high patency rate, although we expected to find that advanced age would be associated with lower patency.

The retrospective nature and single-center design are the main limitations to the present study. Although it is not easy to compare the results of different institutions due to differences in placement techniques, types of catheters used, and patient characteristics, we believe that experienced physicians are associated with improved patient outcomes.

In conclusion, the placement of TDCs in patients should be considered the last resort; however, with the increased use of TDCs and experience, the number of catheter-related complications can be expected to decrease. The prevention of catheter-related complications requires a careful insertion technique and extensive surgical experience. The TDC patency and reducing the number of complications are also closely related to early discharge and the age of patient.

\section{Declaration of conflicting interests}

The authors declared no conflicts of interest with respect to the authorship and/or publication of this article.

\section{Funding}

The authors received no financial support for the research and/or authorship of this article.

\section{REFERENCES}

1. Beigi AA, Sharifi A, Gaheri H, Abdollahi S, Esfahani MA. Placement of long-term hemodialysis catheter (permcath) in patients with end-stage renal disease through external jugular vein. Adv Biomed Res 2014;3:252.

2. Bagul A, Brook NR, Kaushik M, Nicholson ML. Tunnelled catheters for the haemodialysis patient. Eur J Vasc Endovasc Surg 2007;33:105-12.

3. Pereira K, Osiason A, Salsamendi J. Vascular access for placement of tunneled dialysis catheters for hemodialysis: A systematic approach and clinical practice algorithm. J Clin Imaging Sci 2015;5:31.

4. Wang K, Wang P, Liang X, Lu X, Liu Z. Epidemiology of haemodialysis catheter complications: a survey of 865 dialysis patients from 14 haemodialysis centres in Henan province in China. BMJ Open 2015;5:e007136.

5. Ünver S, Atasoyu EM, Evrenkaya TR, Tülbek MY. İki ucu keskin bıçak: Hemodiyaliz kateterleri. Turk J Nephrol 2003;12:184-90.

6. Schummer W, Köditz JA, Schelenz C, Reinhart K, Sakka SG. Pre-procedure ultrasound increases the success and safety of central venous catheterizationt. Br J Anaesth 2014;113:122-9.

7. Aydin Z, Gursu M, Uzun S, Karadag S, Tatli E, Sumnu $\mathrm{A}$, et al. Placement of hemodialysis catheters with a technical, functional, and anatomical viewpoint. Int J Nephrol 2012;2012:302826.

8. Trerotola SO. Hemodialysis catheter placement and management. Radiology 2000;215:651-8.

9. Vascular Access Work Group. Clinical practice guidelines for vascular access. Am J Kidney Dis 2006;48 Suppl 1:S248-73.

10. Allon M. Dialysis catheter-related bacteremia: treatment and prophylaxis. Am J Kidney Dis 2004;44:779-91.

11. Caramori J, Barretti P, Giannini M. Acessos vasculares para hemodiálise. In: Maffei F, editor. Doenças vasculares periféricas. Rio de Janeiro: Medsi.; 2002. p. 1724-36.

12. Miller LM, Clark E, Dipchand C, Hiremath S, Kappel J, Kiaii M, et al. Hemodialysis tunneled catheter-related infections. Can J Kidney Health Dis 2016;3:2054358116669129.

13. National Kidney Foundation. KDOQI Clinical Practice Guidelines and Clinical Practice Recommendations 2006 Updates. Hemodialysis Adequacy, Peritoneal Dialysis Adequacy, Vascular Access. Am J Kidney Dis 2006;48(Suppl 1):S1-S322.

14. Schwab SJ, Beathard G. The hemodialysis catheter conundrum: hate living with them, but can't live without them. Kidney Int 1999;56:1-17.

15. Coentrão L, Santos-Araújo C, Dias C, Neto R, Pestana M. Effects of starting hemodialysis with an arteriovenous fistula or central venous catheter compared with peritoneal dialysis: a retrospective cohort study. BMC Nephrol 2012;13:88.

16. Maki DG, Kluger DM, Crnich CJ. The risk of bloodstream infection in adults with different intravascular devices: a systematic review of 200 published prospective studies. Mayo Clin Proc 2006;81:1159-71. 
17. Lee O, Raque JD, Lee LJ, Wivell W, Block CA, Bettmann MA. Retrospective assessment of risk factors to predict tunneled hemodialysis catheter outcome. J Vasc Interv Radiol 2004;15:457-61.

18. Al-Solaiman Y, Estrada E, Allon M. The spectrum of infections in catheter-dependent hemodialysis patients. Clin J Am Soc Nephrol 2011;6:2247-52.

19. Fry AC, Stratton J, Farrington K, Mahna K, Selvakumar $\mathrm{S}$, Thompson $\mathrm{H}$, et al. Factors affecting long-term survival of tunnelled haemodialysis catheters--a prospective audit of 812 tunnelled catheters. Nephrol Dial Transplant 2008;23:275-81.

20. Lund GB, Trerotola SO, Scheel PF Jr, Savader SJ, Mitchell SE, Venbrux AC, et al. Outcome of tunneled hemodialysis catheters placed by radiologists. Radiology
1996;198:467-72.

21. Shingarev R, Barker-Finkel J, Allon M. Natural history of tunneled dialysis catheters placed for hemodialysis initiation. J Vasc Interv Radiol 2013;24:1289-94.

22. Gołębiowski T, Kusztal M, Letachowicz K, Garcarek J, Porażko T, Penar J, et al. Difficulties with tunneling of the cuffed catheter: a single-centre experience. Sci Rep 2018;8:3314.

23. Salonen TE, Saha H. Structured outpatient peritoneal dialysis catheter insertion is safe and cost-saving. Perit Dial Int 2014;34:612-7.

24. Kocaaslan C, Kehlibar T, Yilmaz M, Mehmetoglu ME, Gunay R, Aldag M, et al. Outcomes of arteriovenous fistula for hemodialysis in octogenarian population. Vascular 2018;26:509-14. 